\title{
Gravitational waves from the Hénon-Heiles system
}

\author{
F. Kokubun* \\ Instituto de Física Teórica, UNESP, Rua Pamplona 145, CEP 01405-900, São Paulo, SP, Brazil \\ and Departamento de Astronomia, IAG-USP, Caixa Postal 9638, CEP 01065-970 São Paulo, SP, Brazil
}

(Received 1 October 1996; published 12 January 1998)

\begin{abstract}
In this work we analyze the emission of gravitational waves from the Hénon-Heiles system. We show the qualitative differences among emission of the gravitational waves from regular and chaotic motions. [S0556-2821(97)01722-0]
\end{abstract}

PACS number(s): 04.30.Db

\section{INTRODUCTION}

Gravitational waves have been known to exist since the early days of general relativity. Einstein himself showed that in the weak field limit we get solutions that obey the wave equation. Despite its importance, at the present time there is only indirect evidence of the reality of gravitational waves (such as the analyzed binary pulsar which shows a decrease in the orbital period due to the emission of gravitational waves [1]). It is expected that with the new generation of gravitational wave detectors under construction, more systems will be observed. In this way, several analyses of the possible sources of gravitational waves have been worked out in recent years in the belief that new detectors may be testing models and theories of gravity, stellar models, galactic dynamics, and so on [2].

In the linear regime of general theory of relativity an important feature of a gravitational wave's emitted power is its dependence on the third-order derivative of the quadrupolar moment. In this case, any system which changes its configuration with time is expected to be a good candidate for a gravitational wave source. Systems suffering catastrophic events are among the best candidates to be observed with gravitational wave detectors and a flurry of work searching for the best candidates to be observed is being done by several groups in the world (see [3] and references therein). However, between these systems, which suffer changes in their configurations, there is one that has not been analyzed yet: a chaotic system. An important characteristic of this system is its high sensitivity to initial conditions and a complicated nonperiodic behavior. In gravitational systems, chaotic behavior was studied with care since the pioneering works of Poincare which analyzed the stability of the Solar System. The three-body problem [4], the tumbling of Saturn's satellite hyperon [5], Hénon-Heiles model of galactic dynamics [6], geodesic motion around black holes [7], Bianchi type-IX cosmological models [8], and others [9] are examples of the chaotic behavior in some gravitational systems. In this work, we consider the emission of gravitational waves from a simple system which shows chaotic behavior: the HénonHeiles system [6]. This system was used for the first time to

\footnotetext{
*Permanent address: Departamento de Física, Fundação Universidade do Rio Grande, Caixa Postal 474, CEP 96201-900, Rio Grande, RS, Brazil.
}

study the dynamics of galactic systems. We know that the emission of gravitational waves from galaxies is negligible to its dynamics. Given this restriction, our main concern in this paper is to show the qualitative differences between gravitational waves from chaotic and regular gravitational systems. In this way, because of its simplicity, the HénonHeiles system is well suited for our purpose.

\section{HÉNON-HEILES SYSTEM}

The Hénon-Heiles system is described by the potential

$$
V(x, y)=m \omega^{2}\left[\frac{x^{2}+y^{2}}{2}+\frac{1}{a}\left(x^{2} y-\frac{y^{3}}{3}\right)\right] .
$$

We have three constants in the above potential: the mass $m$, the frequency $\omega$, and the parameter $a$, which set the length scale of the system (note that it is more common to use the parameter $\lambda \equiv m \omega^{2} / a$ instead of $a$ ). We followed the usual approach, setting $a=\omega=m=1$ in our calculations. An important characteristic of this system is that in the range $E=0-1 / 10$ the dynamics is well behaved, whereas for greater energy the chaoticity of the system increases with energy. At $E=1 / 6$ it is completely chaotic and with $E>1 / 6$ the trajectories are unbounded.

We performed several numerical simulations-using the standard fourth-order Runge-Kutta method-with different energy and initial conditions. The power emitted as a gravitational wave was calculated using the quadrupole formula

$$
-\frac{d E}{d t}=\frac{G}{45 c^{5}} \ddot{Q}_{\alpha \beta}^{2},
$$

with $Q_{\alpha \beta}=\int \mu\left(3 x_{\alpha \beta}-\delta_{\alpha \beta} r^{2}\right) d \vec{r}$. In our case, we considered a pointlike object, and thus $\mu=\delta\left(\vec{r}-\vec{r}_{i}\right)$. Then,

$$
\begin{gathered}
Q_{x x}=2 x^{2}-y^{2}, \\
Q_{y y}=2 y^{2}-x^{2}, \\
Q_{x y}=3 x y,
\end{gathered}
$$

with $x, y$ being solutions of the Hénon-Heiles system. For the sake of simplicity, we assumed that the decrease in the energy carried away as gravitational waves is negligible during the time period considered, thus keeping its energy constant 


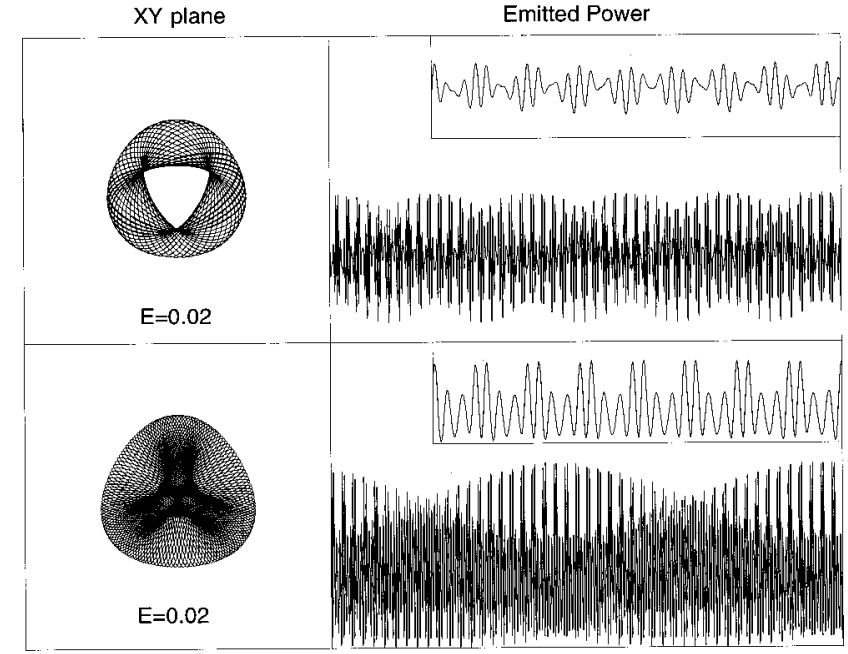

FIG. 1. The power emitted as gravitational waves by particle and its trajectories in the Hénon-Heiles potential with $E=0.020$, for two different initial conditions.

during the integration time. Indeed, assuming that the size of the system is nearly of typical galaxies, the energy carried away as gravitational waves would be very small. Later on in this work we will discuss qualitatively how the dynamics is changed when we consider the energy carried away as gravitational waves.

In Fig. 1 we show the results with $E=0.020$ and using two distinct initial conditions. With this energy, we know that the trajectories are regular. The figure shows the trajectories in the $X Y$ plane (left box) and the power emitted as a gravitational wave (right box). The small rectangle shows a small part of the whole spectrum. Note that the emitted power is periodic, reflecting the characteristics of the trajectory.

Because of the presence of chaotic motions in the HénonHeiles system, it is also important to consider how the spectrum of the emitted power changes in this case. For this purpose, we show in Fig. 2 the case with $E=0.165$ with two different initial conditions. In the bottom graph we have a regular motion and in the upper graph a chaotic motion. When the motion is regular, the emission of gravitational waves is nearly regular and periodic, similar to the previous case. However, in the chaotic case the emission is irregular and shows the presence of irregular peaks in the spectrum. These irregularities are due to its complicated nonperiodic trajectory.

In Table I, we show the statistics of the total energy emitted and the maximum power emitted with $E=0.020$ and $E=0.165$. In this last case a chaotic motion may occur, and thus also we show the same information separating regular motions from chaotic ones. The energy carried away as gravitational waves is greater in the regular case than in the chaotic one at the same energy. However, the maximum emitted power is greater in the chaotic case than the regular one. This difference is due to sudden changes in the trajectories, resulting in a high value of the derivative in its quadrupolar moment. Note that the chaotic case shows several nearly quiescent periods with a relatively small emission of gravitational waves. Because of these periods, the total en-

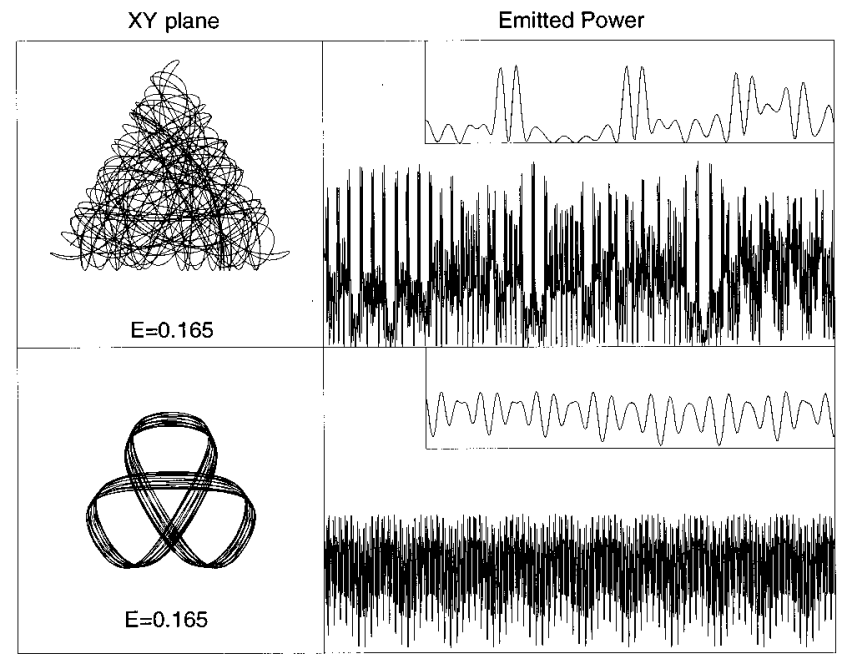

FIG. 2. The power emitted as gravitational waves by particles in the Hénon-Heiles system, with $E=0.165$, for two different initial conditions. We show also the trajectories in the $X Y$ plane.

ergy carried away as gravitational waves is lower than the regular case.

In Fig. 3 we show how the total energy emitted as gravitational waves varies with respect to the initial energy $E$. We also plot the energy emitted when the trajectories are circular, i.e., when the system consists of two bodies orbiting under the action of the Newtonian potential. In this case we know that

$$
\left\langle\frac{d E}{d t}\right\rangle \propto E^{5}
$$

whereas in the Hénon-Heiles case, our numerical simulations resulted in

$$
\left\langle\frac{d E}{d t}\right\rangle \propto E^{1.95}
$$

These graphs are normalized such that total energy emitted when $E=0.01$ is equal in both cases. Thus, the numerical values of $\langle d E / d t\rangle$ are meaningless, only its slope being important. In the Hénon-Heiles system we have an upper limit to the energy if we want a bound system, whereas in the Newtonian case there is not - of course, for very high energy it would be important to consider relativistic effects.

TABLE I. Total energy emitted $\Delta E$ and maximum power $P_{\max }$ when energy is $E=0.165$ and $E=0.020$, with $\sigma=G /\left(45 c^{5}\right)$. In the first case, trajectories may be chaotic. In the table we show the results when considering both chaotic and regular motions, only regular motions, and only chaotic motions.

\begin{tabular}{lccc}
\hline \hline Energy & $\Delta E / \sigma$ & $P_{\max } / \sigma$ & Type of motion \\
\hline \multirow{2}{*}{0.165} & $(2.92 \pm 0.57) \times 10^{3}$ & $14.16 \pm 1.77$ & chaotic + regular \\
& $(3.55 \pm 0.09) \times 10^{3}$ & $11.97 \pm 0.38$ & regular \\
0.020 & $(2.56 \pm 0.37) \times 10^{3}$ & $15.48 \pm 0.19$ & chaotic \\
\hline \hline
\end{tabular}




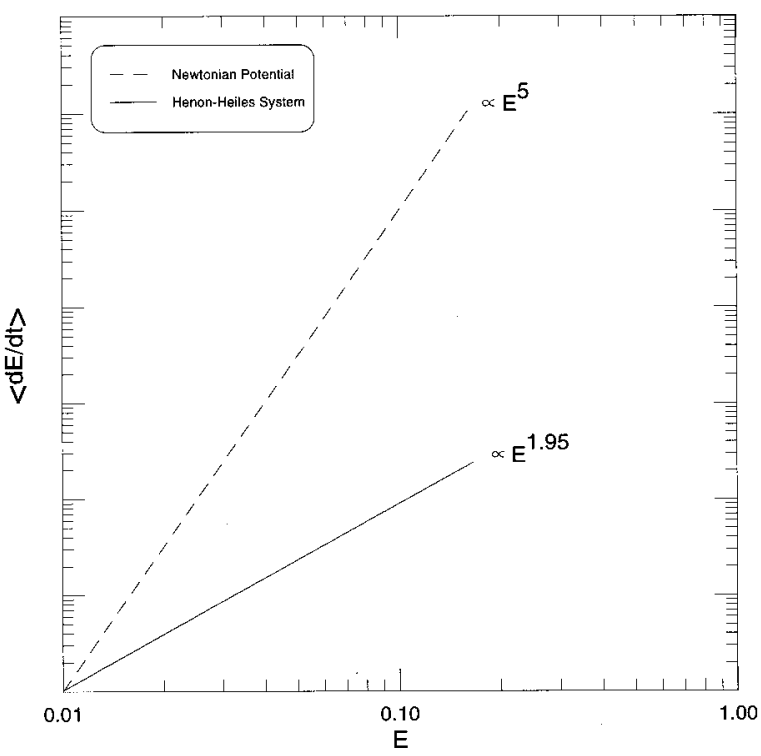

FIG. 3. Mean power emitted as a function of the initial energy with solid line representing the Hénon-Heiles case and dotted line the two bodies in circular orbit in a Newtonian potential. The results are normalized such that at energy $E=0.01$ we set $d E / d t$ equal in both cases.

As we have said before, in a more realistic situation it would be important to consider the energy loss due to the emission of gravitational waves. For this purpose, consider a system initially with $E>1 / 10$. Then its energy decreases with time at a rate which depends on the characteristics of the system (its mass $m$, frequency $\omega$, and length scale $a$ ). However, in all cases after a suitable time interval, the system will lose sufficient energy, reaching a regime with $E<1 / 10$ and becoming a regular one. It is important to remember that chaotic systems are very sensitive to the variations of their initial conditions, and thus during the passage from the $E>1 / 10$ to $E<1 / 10$ state the system may wander from chaotic to regular, resulting in a very complicated dynamics. Given these complications, the final state after a suitable time interval will be a regular state.

\section{CONCLUDING REMARKS}

In this work we have shown that the presence of chaotic motion results in an irregular emission of gravitational waves. However it is important to remember that our model — the Hénon-Heiles potential—is highly idealized, in such a way that our results are not applicable to a realistic astrophysical system. (However, in a recent work of Vieira and Letelier, they show that a black hole system with an external halo has a gravitational potential with terms similar to the Hénon-Heiles system [10].) Given such restrictions, the fact that gravitational waves from chaotic systems show different features, a detailed analysis of a more realistic system will be interesting. Several possible extensions of gravitational waves from chaotic systems may be performed. Note that a highly irregular and peaked spectrum may be a signature of a chaotic motion. However, in some cases these signals may be confused with stochastic noise due to several other sources in the sky. In this way it would be desirable to have adequate data analysis tools to distinguish a stochastic from a chaotic origin of the detected signal. Also, a detailed search of possible realistic astrophysical systems which display chaotic motions with detectable emission of gravitational waves would be desirable.

However, since gravitational waves carry away energy from the system, an initially chaotic system may change its dynamics from this regime to a regular one during its evolution. In this way, it may be important to consider the emission of gravitational waves in the study of long period behavior of a chaotic system, even when gravitational wave emission is negligible. Following this line of reasoning, we are now analyzing the behavior of a restricted three-body problem, which is a paradigm of a gravitational system suffering chaotic motions.

\section{ACKNOWLEDGMENTS}

The author thanks G.E.A. Matsas for comments and suggestions. This work was partially supported by Fapesp, Brazilian Financial Agency.
[1] R. A. Hulse and J. H. Taylor, Astrophys. J. 195, L51 (1975).

[2] K. S. Thorne, in 300 Years of Gravitation, edited by S. W. Hawking and W. Israel (Cambridge University Press, Cambridge, England, 1987); K. S. Thorne, in Proceedings of the Snowmass 94 Summer Study on Particle and Nuclear Astrophysics and Cosmology, edited by E. W. Kolb and R. Peccei (World Scientific, Singapore, 1994).

[3] L. Blanchet, in Proceedings of the Les Houches School, gr-qc/ 9607025 (in press).

[4] See, for example, A. Milani, in Predictability, Stability, and Chaos in N-Body Dynamical Systems, edited by A. E. Roy (Plenum, New York, 1991); R. H. Smith and
V. Szebehely, ibid.

[5] J. Wisdon, Proc. R. Soc. London A413, 109 (1987).

[6] M. Hénon and C. Heiles, Astrophys. J. 69, 73 (1964).

[7] G. Contoupolos, Proc. R. Soc. London A431, 183 (1990); A435, 551 (1991); L. Bombelli and E. Calzetta, Class. Quantum Grav. 9, 2573 (1992).

[8] G. Francisco and G. Matsas, Gen. Relativ. Gravit. 20, 1947 (1988).

[9] D. Hobill, A. Burd, and A. Coley, Deterministic Chaos in General Relativity (Plenum, New York, 1994).

[10] W. M. Vieira and P. S. Letelier, Phys. Rev. Lett. 76, 1409 (1996); 76, 4098 (1996). 